Cahiers $d u$ MONDE RUSSE

\section{Cahiers du monde russe}

Russie - Empire russe - Union soviétique et États indépendants

$59 / 4 \mid 2018$

Varia

\title{
Vladislav Rjeoutski, Quand le français gouvernait la
}

Russie

L'éducation de la noblesse russe, 1750-1880

\section{Wladimir Berelowitch}

\section{OpenEdition}

Journals

Édition électronique

URL : https://journals.openedition.org/monderusse/10611

DOI : 10.4000/monderusse. 10611

ISSN : 1777-5388

Éditeur

Éditions de l'EHESS

\section{Édition imprimée}

Date de publication : 1 octobre 2018

Pagination : $598-601$

ISBN : 978-2-7132-2747-9

ISSN : $1252-6576$

Référence électronique

Wladimir Berelowitch, «Vladislav Rjeoutski, Quand le français gouvernait la Russie », Cahiers du monde russe [En ligne], 59/4 | 2018, mis en ligne le 01 octobre 2018, consulté le 08 janvier 2022. URL : http:// journals.openedition.org/monderusse/10611; DOI : https://doi.org/10.4000/monderusse.10611 
Vladislav RJEOUTSKI, éd., Quand le français gouvernait la Russie L'éducation de la noblesse russe, 1750-1880

Paris : L'Harmattan, 2016, 395 p.

L'ouvrage que nous présentons ici s'inscrit dans un ensemble de publications historiques qui, depuis une dizaine d'années, que ce soit en Russie ou dans les pays occidentaux, renouvellent considérablement l'histoire de l'éducation en Russie, notamment au XVIII ${ }^{\mathrm{e}}$ siècle. Composé par Vladislav Rjéoutski, qui a été pour beaucoup dans ce renouvellement, il sert en quelque sorte de complément au recueil qu'il a publié en 2013 et qui était consacré aux gouverneurs francophones dans l'Europe moderne, principalement en Europe orientale et centrale ${ }^{1}$. Ce nouveau volume se présente comme un ensemble de textes, dont les auteurs sont des gouverneurs ou en tout cas des instituteurs au sens de l'époque, toujours francophones, chargés de l'éducation de la noblesse, ou de la haute noblesse, de l'Empire russe. La période concernée est la seconde moitié du XVIII ${ }^{\mathrm{e}}$ et le début du XIX ${ }^{\mathrm{e}}$ siècle, à l'exception du dernier des douze chapitres, qui est consacré à un enseignant anarchiste, ancien communard, employé dans la famille de Léon Tolstoï.

Les textes sont regroupés en deux parties. La première contient des textes de caractère programmatique et normatif, destinés généralement à l'éducation domestique : plans d'éducation qui se présentent sous ce titre ou bien qui sont développés dans une correspondance, observations d'un gouverneur destinées aux parents, son journal « de bord ", etc. La seconde, qui regroupe des textes de caractère personnel (mémoires, correspondances) est constituée de témoignages émanant des gouverneurs sur les conditions dans lesquelles ils travaillèrent et vécurent dans 
leur milieu d'accueil en Russie. L'ouvrage est précédé d'une longue introduction de V. Rjéoutski et d'introductions généralement plus courtes, en tête de chacun des 12 chapitres : autant d'études de cas, rédigées par les historiens qui présentent les documents, leurs auteurs, les contextes dans lesquels ils ont vu le jour et ont été conservés. Outre V. Rjéoutski lui-même, qui a signé ou cosigné sept de ces introductions, les auteurs sont spécialisés dans l'histoire du XVIII ${ }^{\mathrm{e}}$ siècle : Alexandre Tchoudinov, Vladimir Somov, Natalia Vochtchinskaïa, André Bandelier, Alexandre Stroev, Michel Mervaud, Isabelle de Lassus, auxquels il faut joindre Alla Polossina, spécialiste de Tolstoï.

Presque tous ces documents sont inédits et souvent peu connus ou inconnus, ce qui suffit déjà à en montrer l'intérêt. À l'exception d'un seul chapitre qui contient des extraits très parcellaires du journal du berlinois Paul Birot de Morogues (chapitre 10), il s'agit de documents entiers ou bien, lorsqu'il s'agit de plusieurs documents, ceux-ci font corps et sens. Même dans les cas où la documentation est abondante, comme l'énorme ensemble laissé par Gilbert Romme et ses confrères et admirateurs, gouverneurs des familles Stroganov et Razumovskij, ou bien la correspondance de Frédéric-César de La Harpe, éducateur des grands-ducs Alexandre et Constantin au moment de la Révolution française, les auteurs ont pris soin de choisir des textes entiers, informatifs et mal connus, échappant ainsi à un défaut trop répandu dans ce type de publications, à savoir l'émiettement des extraits, l'anthologie construite sur un principe thématique. Bien au contraire, grâce à ces choix judicieux, grâce aussi aux efforts des historiens qui ont travaillé avec une évidente communauté d'approches et de problématiques, le volume présenté bénéficie d'une grande cohérence de sorte que le lecteur peut avoir l'impression de lire une monographie.

Sans prétendre aborder tous les sujets qui surgissent à la lecture de cet ouvrage, arrêtons-nous sur ceux qui nous paraissent les plus éclairants sur le chapitre de l'éducation.

D'abord les idées. Les plans d'études et les professions de foi pédagogiques qui sont reproduits ici procèdent d'une pratique qui avait cours dans l'Europe de cette époque, et dont on trouve beaucoup d'exemples, souvent restés inédits, en Russie, à deux différences près : dans l'Europe occidentale, le plan d'études développé était le plus souvent destiné soit à des familles royales, car l'éducation y prenait davantage d'importance qu'ailleurs, soit à des établissements tels que le collège, le lycée, le gymnase allemand, etc. Ce genre de publications prit une importance particulière avec le bannissement des jésuites et les projets d' « éducation nationale » (l'expression était française à l'origine, la réalisation surtout germanique) : ainsi le Plan d'éducation publique de l'abbé Coyer en 1770, évoqué par V. Somov. Tandis qu'en Russie, ces plans d'études (règlements ou projets de règlements) qui concernaient aussi des écoles tels que les Corps des cadets, furent, semble-t-il, assez répandus dans les familles, en tout cas dans la haute noblesse. Et aussi, seconde particularité, les plans russes frappent par leur caractère circonstancié, systématique, comme si les rédacteurs ne voulaient rien laisser dans l'ombre et entendaient graver leurs idées et leurs programmes dans le marbre. Ainsi le plan rédigé par « James », gouverneur dans la famille des Razumovskij, et présenté par A. Tchoudinov. Certes son caractère en quelque sorte « philosophique » s'explique en partie par la personnalité de 
son auteur et par l'attachement de celui-ci à Gilbert Romme, déjà cité, qui se trouvait au centre d'une sorte de petite cour pédagogique, à laquelle appartenait aussi Démichel, bien présent dans le volume et gouverneur du cousin de Paul Stroganov. Mais nous retrouvons ce trait dans beaucoup d'autres plans du même type qui, assimilant les leçons de Locke et de Rousseau, reposaient sur l'idée que l'éducation de l'enfant, du jeune homme et à plus forte raison de la jeune fille était plus importante que l'instruction et que le gouverneur était de ce fait même investi d'un rôle capital. Surtout, cette pratique était neuve en Russie, de sorte que les familles des élèves étaient le plus souvent peu informées sur le chapitre de l'éducation tout en étant désireuses d'assurer à leurs rejetons une éducation « digne d'un homme bien né » et c'est pourquoi le texte programmatique prenait tant d'importance. Comme l'écrit V. Rjéoutski dans son introduction, ces textes jettent un éclairage sur des pratiques occidentales qui, nécessitant moins de documentation normative, laissèrent peu de traces dans les archives : les cas russes, pour citer une réflexion orale du regretté Bronislaw Bazcko, deviennent ainsi, pour l'historien, « l'autre bout de la lorgnette», la périphérie qui permet de découvrir le centre (ou l'origine) du transfert.

Ce transfert, les gouverneurs et instituteurs, suisses, français, allemands, mais toujours, dans les deux volumes dirigés par V. Rjéoutski, francophones, en sont les agents. D’origines sociales et géographiques diverses, les spécimens présentés ici partagent certains traits communs. Ils se sentent généralement investis d'une mission qu'on pourrait qualifier de civilisatrice, même si - et c'est un des aspects les plus intéressants de cet ouvrage - leurs programmes sont nécessairement les fruits d'un échange avec les familles d'accueil et subissent une adaptation à chaque cas individuel. Il n'en reste pas moins que cette mission vise l'universalité, celle de l'honnête homme dans son acception la plus large, alors même que la noblesse russe de cette époque se destine massivement la carrière militaire et que les héros de l'ouvrage exercent leurs talents dans des familles de la haute noblesse, les Stroganov, les Golycyn, les Apraksin, les Orlov, etc. Il est frappant, par exemple, que le programme de Le Clerc, un médecin devenu enseignant au Corps des cadets, vise un spectre très large d'occupations, y compris économiques, ce qui intrigue V. Somov dans son introduction. Cependant, l'insistance de Le Clerc sur les ouvrages des physiocrates, qu'il entend faire lire aux élèves, laisse peut-être entrevoir une démarche conjoncturelle puisque la période fut marquée par les encouragements prodigués par Catherine II à la Société libre d'Économie et à l'agriculture (chapitre 4).

Enfin, dernier aspect que nous voudrions souligner et qui fait l'objet de développements tant chez les auteurs de l'ouvrage que chez ceux des textes présentés, les gouverneurs qui font irruption dans la haute société russe ne jouissent pas plus qu'ailleurs en Europe d'un statut social aisément identifiable et c'est ce qui rend leurs témoignages ou leurs correspondances avec leurs employeurs particulièrement précieux. Oscillant entre une considération ou des marques de respect qui miment une égalité sociale utopique et un traitement qui le rabaisse à un statut de domestique libre, le gouverneur ne se trouve à l'aise qu'auprès de ses pairs compatriotes, qu'on pourrait étendre aux bibliothécaires et aux secrétaires, avec lesquels il 
forme un petit milieu ou en tout cas un réseau, et porte un regard tantôt bienveillant, tantôt critique, mais toujours original sur la société russe. Sur le fond de cette « élite des gouverneurs » (A. Tchoudinov, p. 99), celle qui a laissé le plus de traces, il est très difficile de se faire une image tant soit peu précise de tous les obscurs qui furent embauchés à moindres frais par les familles de noblesse moyenne et qui laissèrent, à tort ou à raison, une image déplorable d'aventuriers et de charlatans, du reste partagée par certains gouverneurs « de la haute », une image qui alimenta la xénophobie croissante qu'on observe dans la Russie du début du XIX ${ }^{\mathrm{e}}$ siècle.

Nous n'avons fait qu'évoquer ici quelques aspects d'un livre très riche et passionnant, dont la portée, les intérêts, nous paraissent bien plus larges que ne le suggère le titre et qui, comme le précise du reste son sous-titre, concerne toute la préhistoire de la scolarisation des élites russes, qui fut la grande affaire du XIX ${ }^{\mathrm{e}}$ siècle.

1 - Vladislav Rjéoutski et Alexandre Tchoudinov, éds., Le précepteur francophone en Europe, P. : L'Harmattan, 2013.

Wladimir Berelowitch

CERCEC, CNRS, EHESS, PSL 